\title{
Alternative splicing of HER2: a novel mediator of EGFR TKI resistance
}

\author{
Zachary A. Yochum ${ }^{1,2}$, Liza C. Villaruz ${ }^{1 \wedge}$ \\ ${ }^{1}$ Division of Hematology-Oncology, Department of Medicine, UPMC Hillman Cancer Center, Pittsburgh, PA, USA; ${ }^{2}$ Medical Scientist Training \\ Program, University of Pittsburgh School of Medicine, Pittsburgh, PA, USA \\ Correspondence to: Liza C. Villaruz, MD. UPMC Hillman Cancer Center, 5150 Centre Avenue, Pittsburgh, PA 15232, USA. Email: villaruz@upmc.edu. \\ Provenance and Peer Review: This article was commissioned by the editorial office, Translational Lung Cancer Research. The article did not undergo external \\ peer review. \\ Comment on: Hsu CC, Liao BC, Liao WY, et al. Exon 16-Skipping HER2 as a Novel Mechanism of Osimertinib Resistance in EGFR L858R/T790M- \\ Positive Non-Small Cell Lung Cancer. J Thorac Oncol 2020;15:50-61.
}

Submitted Apr 30, 2020. Accepted for publication May 14, 2020.

doi: $10.21037 /$ tlcr-20-618

View this article at: http://dx.doi.org/10.21037/tlcr-20-618

Over the past three decades, lung cancer has been the leading cause of cancer deaths for both men and women (1). Lung cancer mortality remains high as it is usually diagnosed at a late stage and therapies in the metastatic setting have limited efficacy (1). However, there have been marked advances in the treatment of non-small cell lung cancer (NSCLC), the largest subset of lung cancer, since the introduction of immunotherapy and targeted therapy. When patients with advanced NSCLC are initially diagnosed, they are subtyped based on molecular drivers. While subsets of NSCLC patients with a molecular driver, such as those with KRAS mutations, do not as of yet have an approved targeted therapy, approximately $25 \%$ of NSCLC patients have a commercially available targeted agent $(2,3)$. The largest group with a targetable molecular driver is the approximately $15 \%$ of patients with sensitizing EGFR mutations $(2,3)$.

Multiple Phase III trials have demonstrated that first- and second-generation EGFR TKIs lead to improved objective response rates (ORR) and progression-free survival (PFS) compared with platinum-based chemotherapy $(4,5)$. However, the efficacy of first- and second-generation EGFR TKIs in EGFR-mutant NSCLC is limited by the frequency of de-novo resistance $(\sim 20-30 \%)$ and the inevitability of acquired resistance (5-7). Attempts to establish mechanisms of resistance to first- and second-generation EGFR TKIs have demonstrated that a significant proportion of patients develop secondary EGFR mutations (6). Approximately $50 \%$ of patients acquire the "gatekeeper" T790M EGFR mutation, which renders first- and second-generation EGFR TKIs ineffective as they can no longer access the ATP binding cleft of EGFR $(6,8)$. T790M mutant EGFR remains sensitive to third-generation EGFR TKIs, such as osimertinib, which binds covalently to a cysteine residue (C797) in the ATP binding cleft $(6,8)$. Osimertinib was initially approved as a treatment for patients with acquired T790M EGFR mutations following progression on first-line EGFR TKIs as multiple trials demonstrated osimertinib was superior to chemotherapy in this setting (7). Recently, osimertinib became the recommended first-line therapy for patients with EGFR-mutant NSCLC. The FLAURA trial demonstrated that using osimertinib as a first-line agent resulted in superior PFS and overall survival when compared to first-line erlotinib or gefitinib (9).

Despite improved clinical outcomes, response to firstline osimertinib remains limited by acquired resistance (9). The mechanisms of resistance to first-line osimertinib are not well-established. Early studies have demonstrated that resistance mechanisms appear to be less EGFRdependent, as tertiary EGFR mutations and amplification of wild-type (WT) EGFR only account for resistance in $5-10 \%$ of patients (10-12). The predominant EGFR-

^ ORCID: Zachary A. Yochum: 0000-0001-6483-8089; Liza C. Villaruz: 0000-0001-7456-523X. 
independent mechanism is $M E T$ amplification, accounting for resistance in approximately $15 \%$ of patients (10-12). Unfortunately, the mechanism of resistance in approximately $50 \%$ of patients remains unknown (10-12). Additionally, there are no targeted therapies approved for patients following progression on first-line osimertinib. Immunotherapy in combination with platinum-based chemotherapy may be a viable therapeutic option in this setting as the recent IMpower 150 trial demonstrated that chemo-immunotherapy plus bevacizumab improved PFS and OS when compared to chemotherapy alone in EGFRmutant and $A L K$-positive patients who had progressed on a targeted therapy (13). The IMpower 130 trial failed to demonstrate a clinical benefit for the addition of immunotherapy to chemotherapy without bevacizumab in EGFR-mutant NSCLCs (14). It is important to note that in the large registrational trials evaluating pembrolizumab alone or in combination with chemotherapy in patients with non-squamous NSCLC did not enroll patients with EGFR mutations or $A L K$ translocations (15-17). An ongoing phase III trial, KEYNOTE 789, is specifically aimed at addressing if chemo-immunotherapy is a viable strategy in patients with EGFR-mutant NSCLC resistant to EGFR TKIs.

More recently, investigation into osimertinib resistance mechanisms in NSCLC has identified the HER2 signaling pathway as a driver of resistance. HER2 amplification and mutations are molecular drivers in $5-6 \%$ of all NSCLC patients $(3,18)$. HER2 amplification has also been established as a driver of first- and second-generation EGFR TKI resistance in tumors that lack T790M EGFR mutations (19). Initial studies have identified HER 2 amplification or mutations in approximately $5 \%$ of patients with resistance to first-line osimertinib $(10,11)$. While the HER2 pathway has been implicated in osimertinib resistance, it remains unclear whether additional HER2 aberrations mediate resistance to first-line osimertinib and the validity of HER2 pathways alterations as a therapeutic target in osimertinib resistance.

In a recent report, Hsu and colleagues demonstrated that the exon 16-skipping splice variant of HER2 (HER2D16) is a mediator of osimertinib resistance. Initially, the authors examine the expression of HER2D16 in a patient with metastatic EGFR-mutant NSCLC (20). The patient in the study had known lung lesions, liver metastases, and a malignant pleural effusion following progression on two separate EGFR TKIs (gefitinib and erlotinib) and two different lines of chemotherapy (20). Analysis of the malignant pleural effusion revealed L858R and T790M
EGFR mutations. Osimertinib was subsequently initiated and follow-up revealed that the lung lesion responded to therapy but the liver metastasis progressed. Analysis of cellfree DNA on progression revealed increase in the allelic fraction of HER2D16, HER2 amplification, and loss of T790M EGFR expression (20).

After observing HER2D16 expression in a patient following the development of osimertinib resistance, the authors then examined the role of HER2D16 in mediating osimertinib resistance in vitro. Given previous studies demonstrated that HER2D16 primarily functions as a homodimer in breast cancer (21-23), Hsu et al. investigated whether HER2D16 functions as a homo- or heterodimer in NSCLC cells (20). Interestingly, both in cells with low HER family expression and in cells expressing L858R/ T790M mutant EGFR, HER2D16 has the ability to homodimerize and form heterodimers with EGFR (Figure 1) (20). HER2 and HER2D16 overexpression in cells lacking HER family expression was sufficient to cause ERK activation, however, it was not sufficient to cause increased ERK or AKT signaling in cells expressing L858R/T790M mutant EGFR (20). The inability of HER2 to increase ERK and AKT activation in EGFR-mutant NSCLC cells likely reflects maximal activation of these pathways through constitutively active EGFR.

Interestingly, HER2 and HER2D16 overexpression was sufficient to cause osimertinib resistance in H1975 cells, an L858R/T790M EGFR-mutant NSCLC cell line (20). Additionally, in H1975 cells, HER2D16 overexpression led to a significantly higher osimertinib IC $_{50}$ when compared to HER2 overexpressing and control cells (20). Consistent with previous studies (8), osimertinib suppressed EGFR, ERK, and AKT activation in H1975 cells (20). In HER2 overexpressing cells, osimertinib treatment resulted in suppressed ERK activation and partially suppressed EGFR activation, however, downstream AKT signaling was maintained (20). In HER2D16 overexpressing cells, EGFR, ERK, and AKT activation was maintained in the presence of osimertinib (Figure 1) (20). Additionally, osimertinib did not prevent HER2D16 from forming hetero- or homodimers (20). These data suggest that HER2D16 likely causes osimertinib resistance through persistent activation of EGFR and canonical EGFR signaling pathways.

Previous studies have demonstrated that HER2D16 promotes breast cancer tumorigenesis through activation of SRC $(23,24)$. However, HER2D16 overexpression did not increase total SRC or phospho-SRC expression in H1975 cells (20). Additionally, genetic or pharmacologic inhibition 


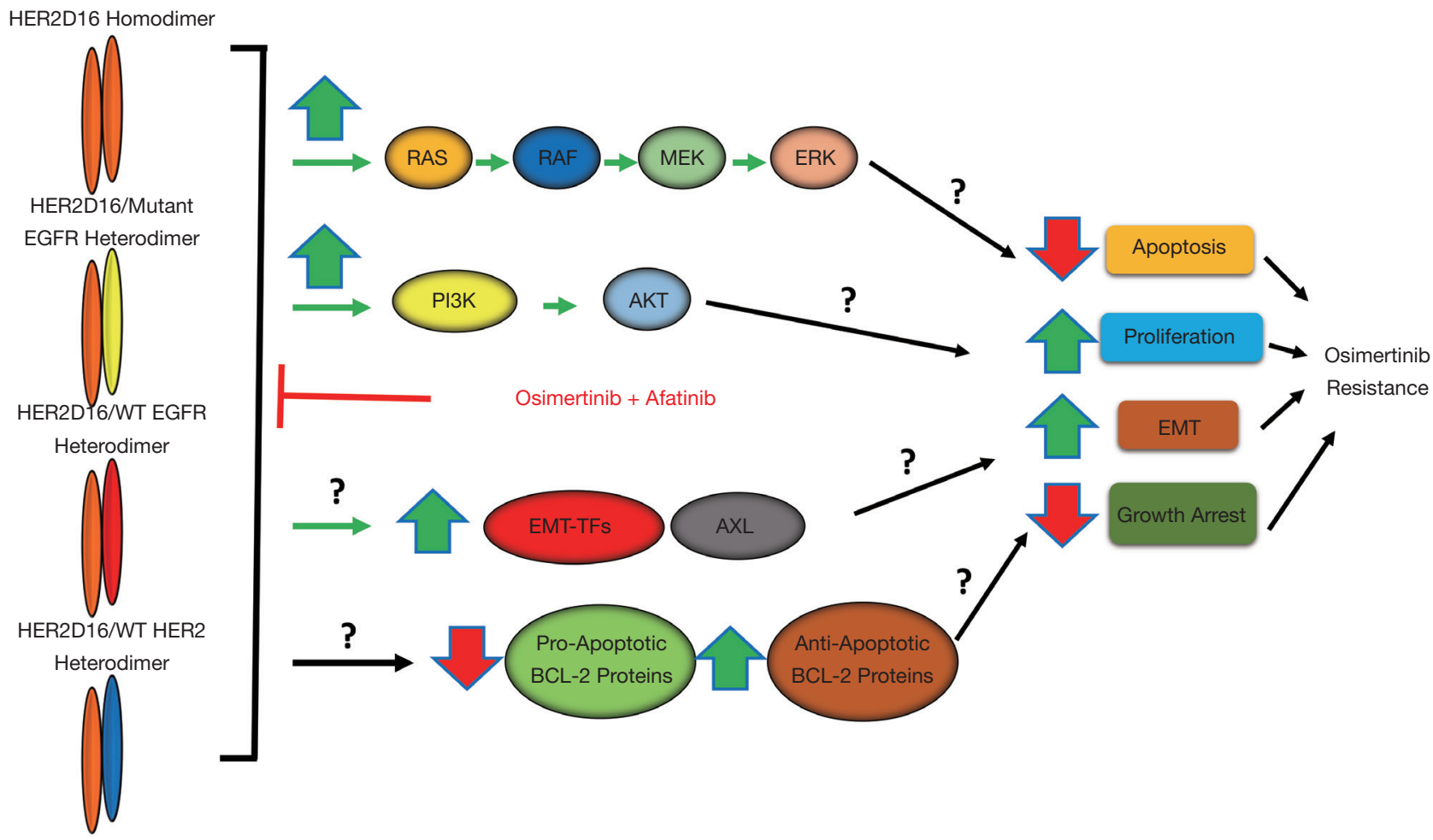

Figure 1 Overexpression of HER2D16, a HER2 exon-16 splice variant, is a novel mediator of osimertinib resistance in EGFR-mutant non-small cell lung cancer (NSCLC) and is associated with reactivation of EGFR pathway signaling. In the discussed report, the authors demonstrate that HER2D16 can homodimerize and/or heterodimerize with mutant EGFR and WT EGFR. Additionally, as HER2D16 expression frequently occurs with HER2 amplification, it may form heterodimers with WT HER2. HER2D16 overexpression was sufficient to cause osimertinib resistance in EGFR-mutant NSCLC cell with a T790M mutation and was associated with reactivation of EGFR and downstream mitogenic/proliferative pathways such as the MAPK and PI3K/AKT pathways. The combination of afatinib, a pan-HER TKI, and osimertinib overcame HER2D16-mediated resistance. Future studies are needed to determine if HER2D16 drives resistance solely through reactivation of MAPK and/or AKT or if other known functions of HER2D16 such as suppression of apoptosis and induction of epithelial-mesenchymal transition also play a role. EMT, epithelial-mesenchymal transition, EMT-TF, epithelial-mesenchymal transitiontranscription factors.

(with dasatinib) of SRC was unable to overcome resistance associated with HER2 or HER2D16 overexpression (20). Interestingly, the use of an irreversible pan-HER inhibitor, afatinib, was able to overcome resistance mediated both by HER2 and HER2D16 overexpression (20). In H1975 cells overexpressing HER2 or HER2D16, the combination of osimertinib and afatinib had synergistic activity that was accompanied by suppression of EGFR and downstream ERK/AKT pathway activation (Figure 1) (20). These results suggest that combining osimertinib with a pan-HER inhibitor may be a strategy to overcome HER2-mediated osimertinib resistance.

The recent report by Hsu et al. is the first study to implicate the Exon-16 skipping HER2 splice variant as a mediator of osimertinib resistance. A strength of this study is that the authors demonstrate HER2D16 expression in an EGFR-mutant NSCLC patient after developing osimertinib resistance. This clinical evidence is accompanied by clear in vitro evidence that HER2D16 overexpression in EGFR-mutant NSCLC cells is sufficient to cause osimertinib resistance (20). The authors also elucidate novel functions of the HER2D16 splice variant in NSCLC cells by demonstrating HER2D16 functions both as a homo- and heterodimer with EGFR and functions to promote therapeutic resistance independently of SRC signaling (20). This differs from the function of HER2D16 in breast cancer. Previous studies demonstrate that in breast cancer HER2D16 promotes tumorigenesis primarily 
as a homodimer and the ability of HER2D16 to mediate therapeutic resistance requires SRC activity (20-24). Studies investigating the factors that influence HER2D12 dimerization and the structure of the HER2 exon-16 skipping splice variant are lacking. Future studies are needed to delineate the structural changes in HER2 caused by exon-16 skipping, structural changes in HER2D16 induced by hetero- versus homodimer formation, and the similarity in kinase domain structure between HER2D16, WT HER2, and WT EGFR. These structural studies are important as they may have therapeutic significance as mutant or aberrant receptor tyrosine kinases (RTKs) can share significant structural overlap with wild-type RTKs. For example, the kinase domain of Exon-20 insertion mutant EGFR shares significant structural similarity to WT EGFR (25). Afatinib, which irreversibly inhibits wildtype EGFR as well as HER2 and HER4, has limited efficacy in the EGFR exon-20 insertion mutant NSCLC population as the structural similarity between the exon-20 insertion mutant EGFR and WT EGFR significantly narrows the therapeutic index of afatinib (25). The dose in vivo required to effectively inhibit the exon-20 insertion mutant EGFR is limited by the side-effects of inhibiting EGFR WT (25).

While the authors provide a possible therapeutic strategy of combining afatinib with osimertinib, to overcome HER2D16-mediated therapeutic resistance, afatinib may have additional side-effects in combination with osimertinib associated with inhibition of WT EGFR. Consequently, paralleling structural studies of HER2D16, in vivo studies are needed to ensure that afatinib in combination with osimertinib is not only efficacious in the setting of HER2D16-driven osimertinib resistance but also can adequately inhibit HER2D16 without significant toxicity or side-effects associated with WT EGFR inhibition. This combination was utilized in a $E G F R$-mutant NSCLC patient who developed a T790M EGFR mutation, HER2 amplification, and a HER2 Exon 20 mutation following progression on icotinib, a first-generation EGFR TKI (26). The combination of osimertinib and afatinib was welltolerated (grade 2 diarrhea and grade $1 \mathrm{rash}$ ) and led to a partial response after 4 weeks of treatment (26). Conversely, a recent study demonstrated that the combination of osimertinib and erlotinib in a transgenic model of EGFRmutant lung cancer was associated with kidney fibrosis and atrophy (27). Future studies should also evaluate osimertinib in combination with other HER2-specific inhibitors with well-established toxicity profiles like trastuzumab. While the evidence is conflicting, HER2D16 may be a marker of HER2 dependence and a predictor of response to trastuzumab $(18,22,23)$. Interestingly, a recent case report identified an activating HER2 mutation, HER2 amplification and a T790M EGFR mutation in a patient with EGFR-mutant NSCLC following progression on gefitinib. The patient received osimertinib and trastuzumab, which resulted in a clinical and molecular response, without overt toxicity (28).

An unanswered question remains as to the relevance of HER2D16 as a mechanism of resistance to first-line osimertinib, and the role of HER2D16 as a mediator of resistance in EGFR-mutant NSCLC that does not harbor T790M mutations. While HER2 amplification and mutations have been observed as a resistance mechanism to first-line osimertinib (11), HER2 exon-16 splice variant expression has not been evaluated in this setting. Initial studies suggest that mechanisms of resistance to firstline osimertinib appear to be more EGFR-independent when compared to second-line osimertinib (10-12). For example, a recent study demonstrated that histological transformation to squamous NSCLC or small cell lung cancer (SCLC) occur in a higher frequency in the setting of first-line osimertinib (12). Additionally, given the presence of HER2D16 expression before the initiation of osimertinib and sequencing data only being available from plasma, it is unclear whether HER2D16 expression was present in the aforementioned patient's lung lesion or liver metastasis before osimertinib treatment. Importantly, the patient's lung lesion responded to osimertinib treatment while the patient's liver metastasis did not (20). The presence of HER2D16 expression in circulating tumor DNA (ctDNA) before osimertinib and the lack of response of the liver lesion suggests that HER2D16 was likely a mechanism of de-novo, rather than acquired, osimertinib resistance in this patient's liver lesion. The heterogeneity that was present in the aforementioned patient's liver and lung lesions stresses the importance of using paired biopsies in evaluating mechanisms of osimertinib resistance. Utilizing paired biopsies from cohorts of patients who have progressed on first-line osimertinib would allow investigators to establish the relevance of HER2D16 expression as mechanism of de-novo and/or acquired resistance. Additionally, obtaining ctDNA pre- and post-treatment would allow for the validation of using ctDNA as a means detecting HER2D16.

While the authors demonstrate that HER2D16 overexpression was associated with persistent EGFR, AKT, and ERK activation, it is unclear if this is the mechanism(s) by which HER2D16 promotes resistance. 
Furthermore, it is unclear if HER2D16 homodimerization and/or heterodimerization with WT HER2, mutant EGFR, and/or WT EGFR is required for osimertinib resistance (Figure 1). Future studies should be aimed at determining whether HER2D16 promotes osimertinib resistance solely through activation of EGFR and/or downstream mitogenic/proliferative signaling pathways or whether HER2D16 promotes resistance through alternative mechanisms (Figure 1). Previous work in breast cancer has revealed that HER2D16 expression promotes epithelial-mesenchymal transition (EMT) and stemness potentially through increased expression of AXL and EMT-transcription factors, such as SNAIL1 and TWIST1 (Figure 1) $(21,23,29)$. Interestingly, EMT is associated with resistance to EGFR TKIs, and overexpression of EMTtranscription factors is both sufficient and required for resistance to first- and third-generation EGFR TKIs in EGFR-mutant NSCLC $(30,31)$. Numerous studies have also demonstrated that suppression of apoptosis, specifically through downregulation of the pro-apoptotic protein BIM, mediates resistance to EGFR TKIs (32). Future studies should evaluate whether HER2D16 suppresses osimertinibinduced apoptosis given previous studies have demonstrated that HER2D16 can lead to tamoxifen resistance in breast cancer cells through upregulation of anti-apoptotic BCL-2 proteins (Figure 1) (33). Determining the mechanism(s) why HER2D16 promotes resistance is important as it may render additional therapeutic targets. For example, tumors that undergo EMT as a mechanism of resistance to EGFR TKIs are more sensitive to BCL-2/BCL-xL inhibitors $(30,31)$.

In summary, Hsu and colleagues demonstrated HER2D16, a HER2 splice variant, is a novel mediator of osimertinib resistance in EGFR-mutant NSCLC. This is the first study to demonstrate HER2D expression in a EGFR-mutant NSCLC patient with osimertinib resistance. They also demonstrated that HER2D16 overexpression is sufficient to mediate osimertinib resistance in EGFRmutant NSCLC cells and that combining afatinib and osimertinib may be a novel therapeutic strategy to overcome HER2D16-mediated resistance. Future studies are needed to validate afatinib and osimertinib as a strategy to overcome HER2D16-mediated resistance in vivo and to determine the frequency of this mechanism in patients with resistance to first-line osimertinib. While osimertinib has benefited patients with EGFR-mutant NSCLC, therapeutic resistance remains inevitable. Chemotherapy-based regimens with the possible addition of immunotherapy are the only currently available options for patients following progression on osimertinib in the absence of an actionable resistance mechanisms. Studies like Hsu and colleagues' recent work aimed at identifying and characterizing novel, potentially actionable, mediators of osimertinib resistance are critical to improving outcomes and sparing toxicity associated with chemotherapy in these patients.

\section{Acknowledgments}

Funding: None.

\section{Footnote}

Conflicts of Interest: Both authors have completed the ICMJE uniform disclosure form (available at http://dx.doi. org/10.21037/tlcr-20-618). LCV receives commercial research grants from Genentech, AstraZenca, Exelixis, Incyte, Merck, Rain, and GlaxoSmithKline and is a paid consultant for Achilles. ZAY has no conflicts of interest to disclose.

Ethical Statement: The authors are accountable for all aspects of the work in ensuring that questions related to the accuracy or integrity of any part of the work are appropriately investigated and resolved.

Open Access Statement: This is an Open Access article distributed in accordance with the Creative Commons Attribution-NonCommercial-NoDerivs 4.0 International License (CC BY-NC-ND 4.0), which permits the noncommercial replication and distribution of the article with the strict proviso that no changes or edits are made and the original work is properly cited (including links to both the formal publication through the relevant DOI and the license). See: https://creativecommons.org/licenses/by-nc-nd/4.0/.

\section{References}

1. Siegel RL, Miller KD, Jemal A. Cancer statistics, 2020. CA Cancer J Clin 2020;70:7-30.

2. Kris MG, Johnson BE, Berry LD, et al. Using Multiplexed Assays of Oncogenic Drivers in Lung Cancers to Select Targeted Drugs. JAMA 2014;311:1998-2006.

3. Cancer Genome Atlas Research Network. Comprehensive molecular profiling of lung adenocarcinoma. Nature 2014;511:543-50.

4. Fukuoka M, Wu YL, Thongprasert S, et al. Biomarker analyses and final overall survival results from a phase III, 
randomized, open-label, first-line study of gefitinib versus carboplatin/paclitaxel in clinically selected patients with advanced non-small-cell lung cancer in Asia (IPASS). J Clin Oncol 2011;29:2866-74.

5. Wu YL, Zhou C, Liam CK, et al. First-line erlotinib versus gemcitabine/cisplatin in patients with advanced EGFR mutation-positive non-small-cell lung cancer: analyses from the phase III, randomized, open-label, ENSURE study. Ann Oncol 2015;26:1883-9.

6. Sequist LV, Waltman BA, Dias-Santagata D, et al. Genotypic and histological evolution of lung cancers acquiring resistance to EGFR inhibitors. Sci Transl Med 2011;3:75ra26.

7. Mok TS, Wu YL, Ahn MJ, et al. Osimertinib or PlatinumPemetrexed in EGFR T790M-Positive Lung Cancer. N Engl J Med 2017;376:629-40.

8. Cross DA, Ashton SE, Ghiorghiu S, et al. AZD9291, an irreversible EGFR TKI, overcomes T790M-mediated resistance to EGFR inhibitors in lung cancer. Cancer Discov 2014;4:1046-61.

9. Soria JC, Ohe Y, Vansteenkiste J, et al. Osimertinib in Untreated EGFR-Mutated Advanced Non-Small-Cell Lung Cancer. N Engl J Med 2018;378:113-25.

10. Ramalingam SS, Cheng $\mathrm{Y}$, Zhou C, et al. Mechanisms of acquired resistance to first-line osimertinib: Preliminary data from the phase III FLAURA study. Ann Oncol 2018;29:viii740.

11. Leonetti A, Sharma S, Minari R, et al. Resistance mechanisms to osimertinib in EGFR-mutated non-small cell lung cancer. Br J Cancer 2019;121:725-37.

12. Schoenfeld AJ, Chan JM, Kubota D, et al. Tumor Analyses Reveal Squamous Transformation and Off-Target Alterations As Early Resistance Mechanisms to First-line Osimertinib in EGFR-Mutant Lung Cancer. Clin Cancer Res 2020;26:2654-63.

13. Socinski MA, Jotte RM, Cappuzzo F, et al. Atezolizumab for First-Line Treatment of Metastatic Nonsquamous NSCLC. N Engl J Med 2018;378:2288-301.

14. Cappuzzo F, McCleod M, Hussein M, et al. IMpower130: Progression-free survival (PFS) and safety analysis from a randomised phase III study of carboplatin + nab-paclitaxel $(\mathrm{CnP})$ with or without atezolizumab (atezo) as first-line (1L) therapy in advanced non-squamous NSCLC. Ann Oncol 2018;29:viii742-3.

15. Reck M, Rodriguez-Abreu D, Robinson AG, et al. Pembrolizumab versus Chemotherapy for PD-L1Positive Non-Small-Cell Lung Cancer. N Engl J Med 2016;375:1823-33.
16. Mok TSK, Wu YL, Kudaba I, et al. Pembrolizumab versus chemotherapy for previously untreated, PD-L1expressing, locally advanced or metastatic non-small-cell lung cancer (KEYNOTE-042): a randomised, open-label, controlled, phase 3 trial. Lancet 2019;393:1819-30.

17. Gandhi L, Rodriguez-Abreu D, Gadgeel S, et al. Pembrolizumab plus Chemotherapy in Metastatic NonSmall-Cell Lung Cancer. N Engl J Med 2018;378:2078-92.

18. Li BT, Ross DS, Aisner DL, et al. HER2 Amplification and HER2 Mutation Are Distinct Molecular Targets in Lung Cancers. J Thorac Oncol 2016;11:414-9.

19. Takezawa K, Pirazzoli V, Arcila ME, et al. HER2 amplification: a potential mechanism of acquired resistance to EGFR inhibition in EGFR-mutant lung cancers that lack the second-site EGFRT790M mutation. Cancer Discov 2012;2:922-33.

20. Hsu CC, Liao BC, Liao WY, et al. Exon 16-Skipping HER2 as a Novel Mechanism of Osimertinib Resistance in EGFR L858R/T790M-Positive Non-Small Cell Lung Cancer. J Thorac Oncol 2020;15:50-61.

21. Alajati A, Sausgruber N, Aceto N, et al. Mammary tumor formation and metastasis evoked by a HER2 splice variant. Cancer Res 2013;73:5320-7.

22. Marchini C, Gabrielli F, Iezzi M, et al. The human splice variant Delta16HER2 induces rapid tumor onset in a reporter transgenic mouse. PLoS One 2011;6:e18727.

23. Mitra D, Brumlik MJ, Okamgba SU, et al. An oncogenic isoform of HER2 associated with locally disseminated breast cancer and trastuzumab resistance. Mol Cancer Ther 2009;8:2152-62.

24. Castagnoli L, Iezzi M, Ghedini GC, et al. Activated d16HER2 homodimers and SRC kinase mediate optimal efficacy for trastuzumab. Cancer Res 2014;74:6248-59.

25. Yasuda H, Park E, Yun CH, et al. Structural, biochemical, and clinical characterization of epidermal growth factor receptor (EGFR) exon 20 insertion mutations in lung cancer. Sci Transl Med 2013;5:216ra177.

26. Zhang P, Nie X, Wang B, et al. Combined therapy with osimertinib and afatinib in a lung adenocarcinoma patient with EGFR T790M mutation and multiple HER2 alterations after resistance to icotinib: A case report. Thorac Cancer 2018;9:1774-7.

27. Starrett JH, Guernet AA, Cuomo ME, et al. Drug sensitivity and allele-specificity of first-line osimertinib resistance EGFR mutations. Cancer Res 2020;80:2017-30.

28. Ralki M, Maes B, Pat K, et al. Triple Trouble: A Case of Multiple Resistance Mechanisms after First Generation EGFR-TKI in NSCLC. Case Rep Oncol 2019;12:625-30. 
29. Goyette M-A, Duhamel S, Aubert L, et al. The Receptor Tyrosine Kinase AXL Is Required at Multiple Steps of the Metastatic Cascade during HER2-Positive Breast Cancer Progression. Cell Reports 2018;23:1476-90.

30. Song KA, Niederst MJ, Lochmann TL, et al. Epithelialto-Mesenchymal Transition Antagonizes Response to Targeted Therapies in Lung Cancer by Suppressing BIM. Clin Cancer Res 2018;24:197.

31. Yochum ZA, Cades J, Wang H, et al. Targeting the EMT transcription factor TWIST1 overcomes resistance to

Cite this article as: Yochum ZA, Villaruz LC. Alternative splicing of HER2: a novel mediator of EGFR TKI resistance. Transl Lung Cancer Res 2020;9(4):1606-1612. doi: 10.21037/tlcr20-618
EGFR inhibitors in EGFR-mutant non-small-cell lung cancer. Oncogene 2019;38:656-70.

32. Costa DB, Halmos B, Kumar A, et al. BIM mediates EGFR tyrosine kinase inhibitor-induced apoptosis in lung cancers with oncogenic EGFR mutations. PLoS Med 2007;4:1669-79; discussion 1680.

33. Cittelly DM, Das PM, Salvo VA, et al. Oncogenic HER2\{Delta\}16 suppresses miR-15a/16 and deregulates BCL-2 to promote endocrine resistance of breast tumors. Carcinogenesis 2010;31:2049-57. 